\title{
BMJ Open Hospital readmission after anterior cruciate ligament reconstruction: protocol for a systematic review and meta-analysis
}

\author{
Long Shao, ${ }^{1} \mathrm{Di} \mathrm{Wu}{ }^{2}$ Jia-Ying $\mathrm{Li}^{3}{ }^{3}$ Xiang-Dong Wu (D) , ${ }^{2,4} \mathrm{Xi}$ Zhou, ${ }^{2}$ \\ Gui-Xing Qiu (D) , ${ }^{2}$ Changqi Luo, ${ }^{4}$ Peng-Cheng Xiao, ${ }^{4}$ Jia-Cheng Liu, ${ }^{4}$ \\ Wei Huang (i) 4
}

To cite: Shao L, Wu D, Li J-Y, et al. Hospital readmission after anterior cruciate ligament reconstruction: protocol for a systematic review and meta-analysis. BMJ Open 2020;10:e037888. doi:10.1136/ bmjopen-2020-037888

- Prepublication history for this paper is available online. To view these files, please visit the journal online (http://dx.doi. org/10.1136/bmjopen-2020037888).

LS and DW are joint first authors.

Received 20 February 2020 Revised 05 September 2020 Accepted 22 September 2020

Check for updates

(C) Author(s) (or their employer(s)) 2020. Re-use permitted under CC BY-NC. No commercial re-use. See rights and permissions. Published by BMJ.

For numbered affiliations see end of article.

Correspondence to

Professor Wei Huang;

drhuangwei68@gmail.com

\section{ABSTRACT}

Introduction Anterior cruciate ligament (ACL) injury is one of the most common injuries of the knee. ACL reconstruction (ACLR) has been widely performed as a safe and effective treatment for ACL injuries. As there is an increasing trend in the incidence of ACL injury, hospital readmission after ACLR has attracted renewed attention for the financial burden to both patients and the healthcare system. However, information about hospital readmission after ACLR remains fragmented. Therefore, we plan to systematically review the literature to investigate the rate of, causes and risk factors for hospital readmission after ACLR, and summarise interventions to reduce hospital readmission. This article is to provide the protocol for an upcoming systematic review and meta-analysis on this important issue.

Methods and analysis Reporting of this protocol follows the Preferred Reporting Items for Systematic Review and Meta-Analysis Protocols (PRISMA-P) checklist. Electronic databases, including PubMed, Embase and the Cochrane Library, will be systematically searched from inception to June 2020. No language restrictions will be applied. Studies will be included if they reported hospital readmission or explored the associated potential causes and risk factors for hospital readmission after ACLR. The primary outcome will be the number and time frame of hospital readmission after ACLR. Secondary outcomes will be reasons for readmission, number and types of complications, risk factors for readmission and preventive measures for readmission after ACLR. Quality assessments will be performed by using the Newcastle-Ottawa Scale (NOS). If possible, study results will be summarised in a forest plot, and heterogeneity will be tested by using the Cochran's $Q$ and $I^{2}$ statistics.

Ethics and dissemination No ethical approval is required because our study is not related to patients or animals. The results will be published in a peer-reviewed journal. PROSPERO registration number CRD42020058624.

\section{INTRODUCTION}

The anterior cruciate ligament (ACL) is a crucial fibrous connective tissue that maintains the dynamic stability of the knee joint. Injury to the ACL is one of the most frequent
Strengths and limitations of this study

- This study will be the first systematic review and meta-analysis that explores the rate of, causes and risk factors for hospital readmission after anterior cruciate ligament reconstruction.

- A comprehensive search strategy developed in consultation with a librarian will be applied to include all eligible studies to present up-to-date evidence.

- This study will provide the highest level of evidence to health providers and policymakers.

- Outcome measures reported in different studies may not be comparable, but we will summarise them transparently for elaboration in this respect.

- This study may be limited by heterogeneity, especially the clinical heterogeneity, which might introduce some bias and decrease the reliability of the conclusion.

and devastating knee injuries, which most commonly occurs during sports or exercise. ${ }^{1}$ Estimates are that more than two million people experience ACL injuries worldwide annually. ${ }^{2}$ Although precise data are not available, approximately 250000 ACL injuries occur annually in the USA. ${ }^{34}$ The ACL injury can lead to a number of problems, including knee pain, instability, joint effusion, reduced functional performance, recreational limitations, increased risk of new knee injuries and impaired quality of life $^{5-8}$ In the long run, ACL injuries are associated with increased risks of clinical sequelae including meniscal tears, chondral lesions and early-onset osteoarthritis. ${ }^{9-11}$ However, the ACL has a limited intrinsic healing capacity due to the lack of vascularisation, ${ }^{1}$ and ACL reconstruction (ACLR) has been widely accepted as a safe and effective treatment option for many patients, which aims to restore the functional stability of 
the knee and allow patients to return to preinjury functionality. ${ }^{12-14}$ So far, it is estimated that approximately 125000 ACLR surgeries are performed each year in the USA, more than 800000 worldwide. ${ }^{121315-17}$ The hospitalisation costs of ACLR surgery ranged from $\$ 5000$ to $\$ 44000$ (depending on the type of reconstruction and severity of the injury), together with the increasing trend in the incidence of ACL injury and ACLR treatment, which would lead to an exponential increase in healthcare costs. ${ }^{18-22}$ In light of these evolving trends, hospital readmission is receiving renewed attention not only for the financial burden to the healthcare system but also for the heavy burden to patients, their families and communities. ${ }^{15} 17$

Unfortunately, although both ACLR surgical techniques and postoperative rehabilitation programmes have significantly improved during the past decades, the hospital readmission continues to occur in $0.7 \%-13.1 \%$ of patients, depending on the patient profile and facility specialty. ${ }^{23-27}$ As such, there is a considerable amount of patients encountered postoperative adverse events associated with readmission after ACLR, suffered significant morbidity, experienced severely impaired quality of life and expended higher costs. ${ }^{23-27}$ Although ACLR accompanies with limited postoperative complications, some of them could become the causes of unplanned hospital readmission. ${ }^{28}$ Immobility and use of a tourniquet during the procedure may lead to venous stasis, and anticoagulants are not routinely used after arthroscopic surgery, so venous thromboembolism (VTE) complications represent a clinical problem, which could account for up to $25 \%$ of the readmissions after ACLR. ${ }^{29}{ }^{30}$ Besides, despite ACLR is performed by applying minimally invasive surgery with an arthroscope and using antibiotics, surgical site infections and wound complications still happen, which represent another common cause of hospital readmission. ${ }^{25} 31$ While other complications, such as knee pain or stiffness and respiratory complications, remain unclear for their role on readmission due to the lack of high-level evidence synthesis in the literature. ${ }^{172426}$ In addition, some studies have further explored the risk factors for hospital readmission after ACLR, including male sex, advanced age, obesity, diabetes, prolonged operative time, operated by low-volume surgeons. ${ }^{1724262729}$ However, to our knowledge, the literature lacks a systematic review of hospital readmission after ACLR.

Considering hospital readmission after ACLR poses a significant problem to both patients and healthcare providers, an increasing number of studies have been focusing on characterising the prevalence, potential causes and risk factors of hospital readmission after ACLR. ${ }^{17}$ However, evidence of hospital readmission following ACLR remains fragmented. Therefore, we decided to conduct a systematic review and meta-analysis to address this issue.

\section{OBJECTIVE}

The proposed systematic review and meta-analysis will aim to identify and synthesise currently available evidence to address the following objectives: (1) to provide up-todate information on the rate of hospital readmission after ACLR; (2) to summarise the causes and related risk factors of the hospital readmission after ACLR and (3) to summarise interventions to reduce hospital readmission following ACLR.

\section{METHODS AND ANALYSIS}

\section{Registration information}

This study protocol will be amended and updated in conjunction with the Preferred Reporting Items for Systematic Review and Meta-Analysis Protocols (PRISMA-P) ${ }^{32}$ This systematic review and meta-analysis was prospectively registered in the International Prospective Register of Systematic Reviews. Our systematic reviews and metaanalysis will be conducted and reported in accordance with the guidelines from Meta-analysis of Observational Studies in Epidemiology (MOOSE) and based on a modification of the Cochrane handbook for systematic reviews of interventions. ${ }^{33}$ The completed review will be reported following the Preferred Reporting Items for Systematic Reviews and Meta-Analyses (PRISMA) guidelines. ${ }^{34}$

\section{Eligibility criteria}

Types of studies

Studies that reported hospital readmission or explored the associated causes and risk factors for hospital readmission after ACLR will be potentially eligible, which mainly including prospective cohort studies, retrospective cohort studies, case-control studies and cross-sectional studies. There will be no specific restrictions on the study design, and eligible clinical trials will also be included, but most of the studies are expected to be retrospective observational studies. However, conference abstracts with incomplete data, animal studies, case reports, reviews, letters to the editor and editorial materials will be excluded. There will be no restrictions regarding publication status or language.

\section{Types of participants}

Patients who were admitted to the hospital for an ACLR procedure would be eligible, regardless of whether inpatient-only procedures, outpatient-only procedures or both inpatient and outpatient procedures. We will include children, teenagers and adult patients without the limitation of age, since there is an increasing incidence of ACLR in the young population. Notably, we will include all kinds of ACLR procedures regardless of surgical approaches, ACL grafts and surgical concepts (e.g., single bundle, double bundle). Also, to enhance the clinical significance of this study, both primary ACLR and ACLR revision surgery will be included. In particular, as many meniscal tears are associated with ACL injuries, we will not exclude ACLR with simultaneous meniscal 
treatment. Additionally, there will be no specific restrictions on the time frame and causes of hospital readmission. That is to say, studies focused on either short-term (within 1year after surgery) or long-term (more than 1 year after surgery) readmissions after ACLR, no matter caused by infection, resurgery (e.g., caused by secondary or tertiary ACLR revision, or total knee replacement) or other causes, would be potentially eligible. However, studies will be excluded if patients suffered from multiligament knee injuries and received multiligament reconstruction.

\section{Types of outcome measures}

Our primary outcome measure of interest will be the number and time frame of hospital readmission after ACLR. Secondary outcome measures of interest will be reasons for readmission, number and types of complications, risk factors for readmission and preventive measures for hospital readmission following ACLR.

\section{Patient and public involvement}

In this study, data will not be collected directly from patients; instead, data will be obtained from the published literature and online databases. Therefore, patients will not be involved in the completion of this systematic review protocol or subsequent research.

\section{Search strategy}

We will search the following electronic bibliographic databases from inception to June 2020 to capture all recent relevant studies: PubMed, Embase and the Cochrane Library (Cochrane Database of Systematic Reviews). The search strategy has been developed by two authors (XW and J-YL) with assistance from an external research librarian, which will be tailored to each database using keywords and text terms, such as 'readmission' and 'anterior cruciate ligament reconstruction'. The detailed search strategies are shown in table 1 . Besides, we will search the clinical trial registries, academic dissertations, research conference proceedings and non-traditional publications to minimise publication bias in our data. To ensure the literature saturation, a supplementary search will be conducted using Google Scholar, which might capture eligible studies not returned by other databases. Bibliographies of all eligible studies, relevant systematic reviews and meta-analyses and narrative reviews identified through the initial search will be further screened by hand. No language or publication period restrictions will be applied.

\section{Study selection}

The results of the literature searches will be imported into EndNote V.X8 bibliographic software (Clarivate Analytics, Carlsbad, California, USA) for records management. First, duplicate records will be removed. Second, two reviewers (XW and J-YL) will independently screen the titles and abstracts according to the eligibility criteria. They will develop a screening form and label each record as relevant, irrelevant or indeterminate, and remove the

\begin{tabular}{|c|c|c|}
\hline & Search & Query \\
\hline \multicolumn{3}{|l|}{ PubMed } \\
\hline & $\# 1$ & "Patient Readmission"(Mesh) \\
\hline & \#2 & Readmission(Title/Abstract) \\
\hline & \#3 & Re-admission(Title/Abstract) \\
\hline & $\# 4$ & Readmission*(Title/Abstract) \\
\hline & $\# 5$ & Readmit*(Title/Abstract) \\
\hline & \#6 & Re-admit*(Title/Abstract) \\
\hline & $\# 7$ & Rehospitalization(Title/Abstract) \\
\hline & \#8 & Rehospital ${ }^{\star}($ Title/Abstract) \\
\hline & $\# 9$ & Re-hospital*(Title/Abstract) \\
\hline & \#10 & “Reoperation”(Mesh) \\
\hline & $\# 11$ & Reoperation(Title/Abstract) \\
\hline & \#12 & Re-operation(Title/Abstract) \\
\hline & \#13 & Resurgery(Title/Abstract) \\
\hline & \#14 & Re-surgery(Title/Abstract) \\
\hline & \#15 & "Infections"(Mesh) \\
\hline & \#16 & "Wound Infection"(Mesh) \\
\hline & $\# 17$ & "Surgical Wound Infection"(Mesh) \\
\hline & \#18 & Infection*(Title/Abstract) \\
\hline & \#19 & Wound Infection(Title/Abstract) \\
\hline & \#20 & Surgical Wound Infection(Title/Abstract) \\
\hline & \#21 & Graft failure(Title/Abstract) \\
\hline & \#22 & Graft rupture(Title/Abstract) \\
\hline & \#23 & Reinjury(Title/Abstract) \\
\hline & \#24 & Re-injury(Title/Abstract) \\
\hline & \#25 & $\begin{array}{l}\# 1 \text { OR \#2 OR \#3 OR \#4 OR \#5 OR \#6 OR } \\
\# 7 \text { OR \#8 OR \#9 OR \#10 OR \#11 OR \#12 } \\
\text { OR \#13 OR \#14 OR \#15 OR \#16 OR \#17 } \\
\text { OR \#18 OR \#19 OR \#20 OR \#21 OR \#22 } \\
\text { OR \#23 OR \#24 }\end{array}$ \\
\hline & \#26 & “Anterior Cruciate Ligament”(Mesh) \\
\hline & \#27 & $\begin{array}{l}\text { "Anterior Cruciate Ligament } \\
\text { Reconstruction"(Mesh) }\end{array}$ \\
\hline & \#28 & Anterior Cruciate Ligament(Title/Abstract) \\
\hline & \#29 & ACL(Title/Abstract) \\
\hline & \#30 & \#26 OR \#27 OR \#28 OR \#29 \\
\hline & \#31 & \#25 AND \#30 \\
\hline \multicolumn{3}{|l|}{ Embase } \\
\hline & $\# 1$ & 'hospital readmission'/exp \\
\hline & \#2 & readmission:ti,ab,kw \\
\hline & \#3 & re-admission:ti,ab,kw \\
\hline & \#4 & readmission*:ti,ab,kw \\
\hline & \#5 & readmit*:ti,ab,kw \\
\hline & \#6 & re-admit*:ti,ab,kw \\
\hline & \#7 & rehospitalization:ti,ab,kw \\
\hline & \#8 & rehospital*:ti,ab,kw \\
\hline & $\# 9$ & re-hospital*:ti,ab,kw \\
\hline
\end{tabular}




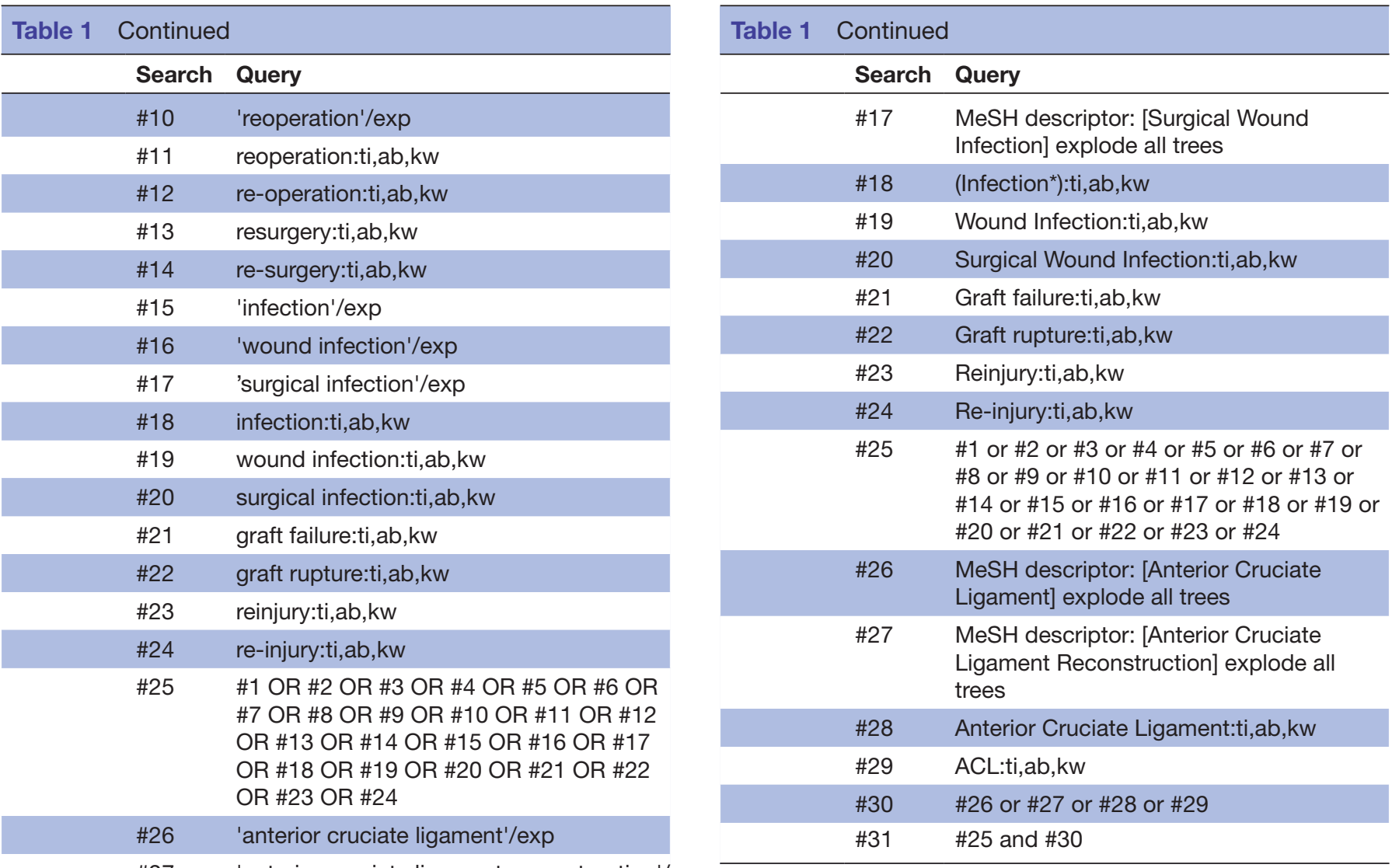

\#27 'anterior cruciate ligament reconstruction'/ exp

\begin{tabular}{|c|c|c|}
\hline & \#28 & 'anterior cruciate ligament':ti,ab,kw \\
\hline & \#29 & acl:ti,ab,kw \\
\hline & \#30 & \#26 OR \#27 OR \#28 OR \#29 \\
\hline & \#31 & \#25 AND \#30 \\
\hline \multicolumn{3}{|l|}{ Cochrane } \\
\hline & $\# 1$ & $\begin{array}{l}\text { MeSH descriptor: [Patient Readmission] } \\
\text { explode all trees }\end{array}$ \\
\hline & \#2 & Readmission:ti,ab,kw \\
\hline & \#3 & Re-admission:ti,ab,kw \\
\hline & \#4 & Readmission*:ti,ab,kw \\
\hline & \#5 & Readmit*:ti,ab,kw \\
\hline & \#6 & Re-admit*:ti,ab,kw \\
\hline & \#7 & Rehospitalization:ti,ab,kw \\
\hline & \#8 & Rehospital*:ti,ab,kw \\
\hline & $\# 9$ & Re-hospital*:ti,ab,kw \\
\hline & \#10 & $\begin{array}{l}\text { MeSH descriptor: [Reoperation] explode } \\
\text { all trees }\end{array}$ \\
\hline & $\# 11$ & Reoperation:ti,ab,kw \\
\hline & \#12 & Re-operation:ti,ab,kw \\
\hline & $\# 13$ & Resurgery:ti,ab,kw \\
\hline & \#14 & Re-surgery:ti,ab,kw \\
\hline & \#15 & $\begin{array}{l}\text { MeSH descriptor: [Infections] explode all } \\
\text { trees }\end{array}$ \\
\hline & \#16 & $\begin{array}{l}\text { MeSH descriptor: [Wound Infection] } \\
\text { explode all trees }\end{array}$ \\
\hline
\end{tabular}

records that did not fulfil the eligibility criteria. Third, the full text of the potential eligible records will be obtained and analysed by two reviewers. The inter-rater agreement will be established using Cohen's kappa statistic, and an $80 \%$ or higher level of agreement is desirable. ${ }^{35}$ Selection discrepancies will be resolved by discussion and involvement of a third reviewer $(\mathrm{WH})$, if necessary. We will record the reasons for exclusion during the process of full-text review. The study selection process will be reported using a PRISMA flow diagram (figure 1).

\section{Quality assessment}

The Newcastle-Ottawa Scale (NOS) will be used to evaluate the methodological quality of the included studies, which offers quality assessment scales for both cohort studies and case-control studies. ${ }^{36}$ This nine-point scale is divided into three major components with a maximum number of items for each category: the selection of the study groups (0-4 points), comparability of the groups (0-2 points) ascertainment of exposure or outcome of interest ( $0-3$ points). In the NOS scale, studies could be awarded one point for each item within selection and exposure categories, and up to two points for items in the comparability category. Furthermore, a higher score represents better methodological quality as well as a lower risk of bias. Studies will be considered to be of high quality if they score $\geq 7$ and low quality if they score $<7$. Two raters (CL and P-CX) will independently apply the scale to each included study, and discrepancies will be resolved by discussion or consultation with a third reviewer (LS) if 


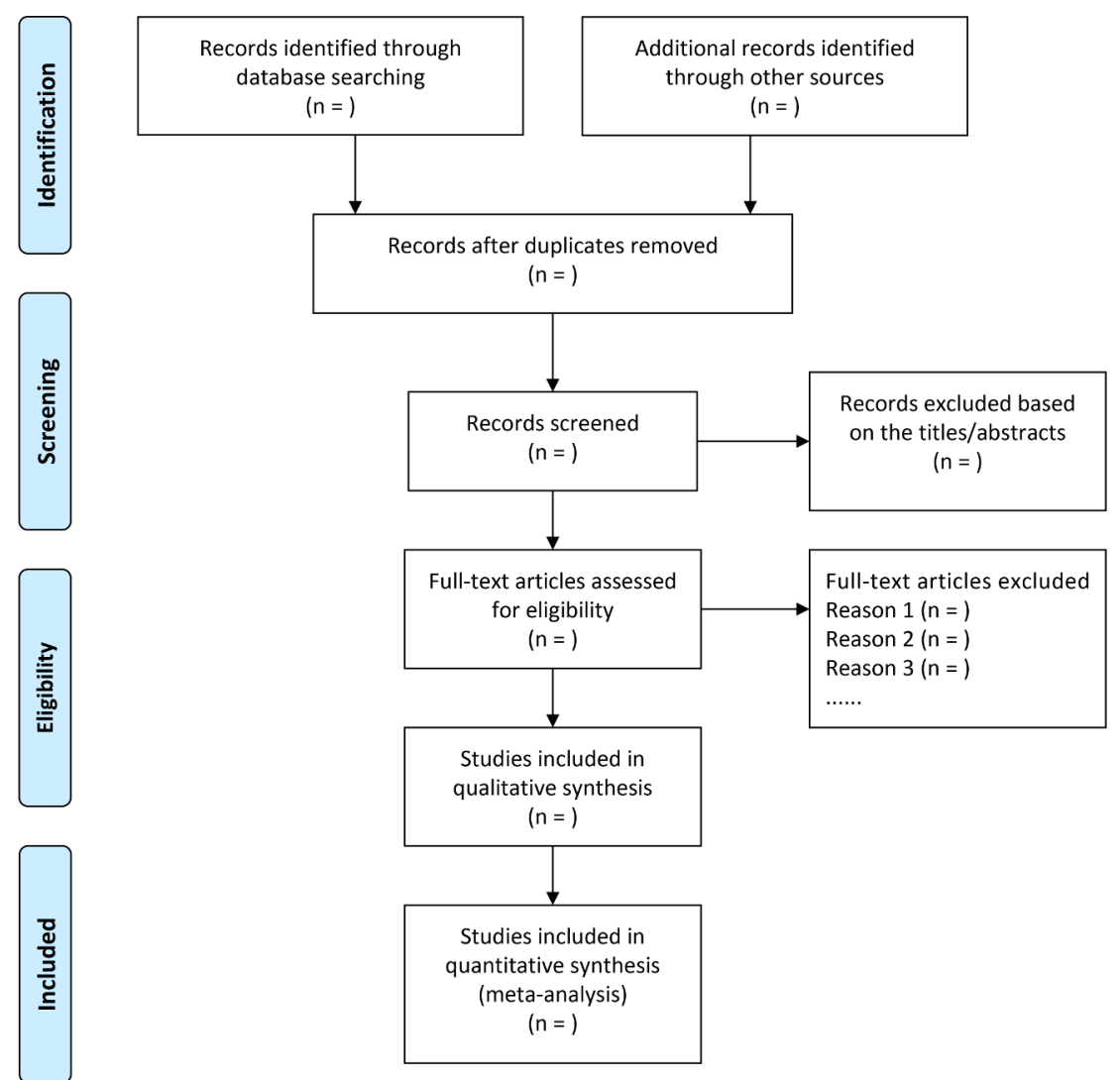

Figure 1 PRISMA flow diagram showing the process of literature screening, study selection and reasons for study exclusion. The PRISMA statement is used worldwide to improve the reporting of systematic reviews and meta-analyses. PRISMA, Preferred Reporting Items for Systematic Reviews and Meta-Analyses.

an agreement could not be reached. Moreover, if other types of studies were included, the appropriate quality assessment tools (e.g., Cochrane risk of bias tool for randomised controlled trials) will be used accordingly.

\section{Data extraction and management}

Two reviewers (DW and XZ) will manually and independently extract data from each included study and insert extracted data into a predefined standardised Microsoft Excel spreadsheet (Microsoft Corporation, Washington, USA). Disagreements will be resolved by discussion and consultation with a third reviewer (G-XQ). The following information will be collected: first author, publication year, country where the study was carried out, study design, study period, the database type, number of patients, patient characteristics, surgical procedure, the definition and time frame of readmission (e.g., 30-day readmission, 90-day readmission, 1-year readmission), number of readmissions, causes of readmission (e.g., allcause, VTE, infection) and how they were identified (e.g., International Classification of Diseases, Ninth Revision, Clinical Modification), number and types of complications (e.g., wound infection), risk factors for readmission and preventive measures for readmission (e.g., complete rehabilitation programmes, pass return to sport criteria). If data were presented as graphs or figures only, Plot Digitizer software would be used to extract data. ${ }^{3738}$ We will further examine the supplementary materials of the included studies and contact corresponding authors to verify the extracted data and request the missing data.

\section{Data synthesis}

A narrative synthesis of the studies included in this review will be provided and structured around the outcomes. If sufficient data available, the outcome data will be further pooled and reported in a forest plot to summarise the results of each study visually. Pooled estimates, with accompanying 95\% confidence intervals (CIs), on readmission rates will be calculated using the DerSimonian and Laird random effects model ${ }^{32}$ For the meta-analysis of the causes (e.g., VTE, wound infection) of hospital readmissions, risk ratios (RRs) with 95\% CIs with the Mantel-Haenszel method will be used for analysis. For the meta-analysis of risk factors associated with hospital readmission, the odds ratios (ORs) with accompanying 95\% CIs will be reported if more than two studies reported that risk factor. The reported RR, univariable OR or univariable HR for each risk variable will be pooled and analysed individually using random-effect models weighted by inverse variance. ${ }^{39}$ For the preventive intervention for hospital readmission, we will qualitatively synthesise the findings from the included studies.

Heterogeneity across studies will be assessed by using the $Q$ statistic with its $p$ value and $\mathrm{I}^{2}$ statistic. The $\mathrm{I}^{2}$ statistic is used to quantify the proportion of total variation in the effect estimation as differences between study results. An 
$\mathrm{I}^{2}$ value of $0 \%$ indicates no observed heterogeneity, $25 \%$ low heterogeneity, $50 \%$ moderate heterogeneity and $75 \%$ high heterogeneity. The included studies are assumed to be heterogeneous accounting for clinical heterogeneity; therefore, a random effects model will be used a priori. ${ }^{3540} \mathrm{~A}$ two-sided $\mathrm{p}$ value $<0.05$ will be considered as statistically significant.

To assess the publication bias, we will examine a funnel plot for asymmetry only when at least 10 studies are included in the meta-analysis. ${ }^{41}$ We will further assess the publication bias according to the Egger regression asymmetry test, and the Begg and Mazumdar adjusted rank correlation tests. ${ }^{42} 43$

\section{Subgroup analysis and sensitivity analysis}

If the number of included studies is sufficient, subgroup analyses and meta-regression analyses will be conducted (e.g., on the risk of bias scores, follow-up periods) to investigate the underlying source of heterogeneity. Sensitivity analysis will be performed by sequential removal of individual studies from the analysis. A value of $0.5 \%$ is set as the a priori threshold, which means if removing a study leads to the hospital readmission rate changed by $0.5 \%$ or more, this will be considered clinically significant. All the above analyses will be performed using Stata statistical software V.14.0 (StataCorp, College Station, Texas, USA).

\section{DISCUSSION}

Hospital readmission rates have been recognised as an essential indicator of patient clinical outcome and healthcare system performance. ${ }^{44}$ Hospital readmissions after ACLR are always associated with adverse postoperative outcomes and numerous publications have investigated the causes for readmission. ${ }^{17}{ }^{24-27}$ The findings indicated that in the short term, readmissions are often attributed to certain medical complications (e.g., infection) or surgical complications (e.g., stiffness of the knee joint) $;^{1724-27}$ while in the long term, readmissions are usually caused by the ACL graft failure and degenerative knee osteoarthritis, which necessitate subsequent operations like ACLR revision surgery and total knee replacement. ${ }^{4546}$ Though previous studies have identified some causes and related risk factors for readmission after ACLR, there remains a lack of synthesis of the current evidence. Therefore, we believe this proposed systematic review and meta-analysis is feasible, attainable and timely.

This systematic review and meta-analysis will provide up-to-date evidence on the rate of, causes and risk factors of hospital readmission after the ACLR. This study will also identify interventions that will be beneficial to ACLR surgeries, perioperative care and postoperative rehabilitation, which will contribute to optimising the operating systems and practices to aid in same-day discharges following ACLR, not only reducing the hospital readmission rate, but also help to relieve pressure from the readmission for both patients and the healthcare providers.
In summary, this review will integrate the existing studies about hospital readmission after ACLR.

\section{Ethics and dissemination}

Ethics approval will not be required for the study, as it bases on previously published data and will not involve in the privacy of any individual patients. The results will be published in a peer-reviewed international journal for dissemination.

\section{Author affiliations}

${ }^{1}$ Department of Orthopaedic Surgery, Ningbo No.6 Hospital, Ningbo, Zhejiang, China ${ }^{2}$ Department of Orthopaedic Surgery, Peking Union Medical College Hospital, Chinese Academy of Medical Sciences \& Peking Union Medical College, Beijing, China

${ }^{3}$ Departments of Nephrology, Peking Union Medical College Hospital, Chinese Academy of Medical Sciences \& Peking Union Medical College, Beijing, China ${ }^{4}$ Department of Orthopaedic Surgery, The First Affiliated Hospital of Chongqing Medical University, Chongqing, China

Acknowledgements The authors thank professor Yong Liu (from the Department of Orthopaedic Surgery, Peking Union Medical College Hospital) for his substantial contributions to the drafting of this protocol.

Contributors LS, X-DW, WH and G-XQ conceived and designed the study. LS and $X$-DW drafted the protocol. J-YL, DW, XZ, LS, CL, P-CX, J-CL, WH and G-XQ revised the protocol. X-DW and J-YL will search for and select eligible studies. DW and XZ will extract the data, LS will check the data. CL and P-CX will assess the risk of bias. J-CL, WH and G-XQ will perform the data synthesis. LS and X-DW act as guarantors of the protocol. All authors approved the publication of the protocol.

Funding The authors have not declared a specific grant for this research from any funding agency in the public, commercial or not-for-profit sectors.

Competing interests None declared.

Patient and public involvement Patients and/or the public were not involved in the design, or conduct, or reporting, or dissemination plans of this research.

Patient consent for publication Not required.

Provenance and peer review Not commissioned; externally peer reviewed.

Open access This is an open access article distributed in accordance with the Creative Commons Attribution Non Commercial (CC BY-NC 4.0) license, which permits others to distribute, remix, adapt, build upon this work non-commercially, and license their derivative works on different terms, provided the original work is properly cited, appropriate credit is given, any changes made indicated, and the use is non-commercial. See: http://creativecommons.org/licenses/by-nc/4.0/.

\section{ORCID iDs}

Xiang-Dong Wu http://orcid.org/0000-0002-3920-4372

Gui-Xing Qiu http://orcid.org/0000-0001-7137-1843

Wei Huang http://orcid.org/0000-0002-8894-0982

\section{REFERENCES}

1 Kiapour AM, Murray MM. Basic science of anterior cruciate ligament injury and repair. Bone Joint Res 2014;3:20-31.

2 Samuelsson K. Anatomic ACL reconstruction-current evidence and future directions. PhD thesis, Göteborg University, Sweden, 2012.

3 Davarinos N, O'Neill BJ, Curtin W. A brief history of anterior cruciate ligament reconstruction. Advances in Orthopedic Surgery 2014;2014:1-6.

4 Musahl V, Karlsson J. Anterior cruciate ligament tear. N Engl J Med 2019;380:2341-8.

5 Faude O, Junge A, Kindermann W, et al. Risk factors for injuries in elite female soccer players. Br J Sports Med 2006;40:785-90.

6 Waldén M, Hägglund M, Ekstrand J. High risk of new knee injury in elite footballers with previous anterior cruciate ligament injury. $\mathrm{Br} J$ Sports Med 2006;40:158-62.

7 Filbay SR, Culvenor AG, Ackerman IN, et al. Quality of life in anterior cruciate ligament-deficient individuals: a systematic review and meta-analysis. Br J Sports Med 2015;49:1033-41. 
8 Filbay SR, Ackerman IN, Russell TG, et al. Health-Related quality of life after anterior cruciate ligament reconstruction: a systematic review. Am J Sports Med 2014;42:1247-55.

9 Lohmander LS, Östenberg A, Englund M, et al. High prevalence of knee osteoarthritis, pain, and functional limitations in female soccer players twelve years after anterior cruciate ligament injury. Arthritis Rheum 2004;50:3145-52.

10 von Porat A, Roos EM, Roos H. High prevalence of osteoarthritis 14 years after an anterior cruciate ligament tear in male soccer players: a study of radiographic and patient relevant outcomes. Ann Rheum Dis 2004;63:269-73.

11 Øiestad BE, Engebretsen L, Storheim K, et al. Winner of the 2008 systematic review competition: knee osteoarthritis after anterior cruciate ligament injury. Am J Sports Med 2009;37:1434-43.

12 Lai CCH, Ardern CL, Feller JA, et al. Eighty-three per cent of elite athletes return to preinjury sport after anterior cruciate ligament reconstruction: a systematic review with meta-analysis of return to sport rates, graft rupture rates and performance outcomes. $\mathrm{Br} J$ Sports Med 2018;52:128-38.

13 Kay J, Memon M, Marx RG, et al. Over $90 \%$ of children and adolescents return to sport after anterior cruciate ligament reconstruction: a systematic review and meta-analysis. Knee Surg Sports Traumatol Arthrosc 2018;26:1019-36.

14 O'Connor RF, King E, Richter C, et al. No relationship between strength and power scores and anterior cruciate ligament return to sport after injury scale 9 months after anterior cruciate ligament reconstruction. Am J Sports Med 2020;48:78-84.

15 Horwitz L, Partovian C, Lin Z, et al. Hospital-Wide all-cause unplanned readmission measure. Baltimore, MD: Centers for Medicare \& Medicaid Services, 2012.

16 MOON Knee Group, Spindler KP, Huston LJ, et al. Ten-Year outcomes and risk factors after anterior cruciate ligament reconstruction: a moon longitudinal prospective cohort study. Am J Sports Med 2018;46:815-25.

17 Cooper JD, Lorenzana DJ, Heckmann N, et al. The effect of obesity on operative times and 30-day readmissions after anterior cruciate ligament reconstruction. Arthroscopy 2019;35:121-9.

18 Herzog MM, Marshall SW, Lund JL, et al. Trends in incidence of ACL reconstruction and concomitant procedures among commercially insured individuals in the United States, 2002-2014. Sports Health 2018;10:523-31.

19 Mall NA, Chalmers PN, Moric M, et al. Incidence and trends of anterior cruciate ligament reconstruction in the United States. Am J Sports Med 2014;42:2363-70.

20 Nagda SH, Altobelli GG, Bowdry KA, et al. Cost analysis of outpatient anterior cruciate ligament reconstruction: autograft versus allograft. Clin Orthop Relat Res 2010;468:1418-22.

21 Bates NA, Hewett TE. Motion analysis and the anterior cruciate ligament: classification of injury risk. J Knee Surg 2016;29:117-25.

22 Ferrari D, Lopes TJA, França PFA, et al. Outpatient versus inpatient anterior cruciate ligament reconstruction: a systematic review with meta-analysis. Knee 2017;24:197-206.

23 Qin C, Helfrich MM, Curtis DM, et al. The effect of surgical setting on anterior cruciate ligament reconstruction outcomes. Phys Sportsmed 2019;47:411-5.

24 Lyman S, Koulouvaris P, Sherman S, et al. Epidemiology of anterior cruciate ligament reconstruction: trends, readmissions, and subsequent knee surgery. J Bone Joint Surg Am 2009;91:2321-8.

25 Jameson SS, Dowen D, James P, et al. Complications following anterior cruciate ligament reconstruction in the English NHS. Knee 2012;19:14-19.

26 Boddapati V, Fu MC, Nwachukwu BU, et al. Procedure length is independently associated with overnight hospital stay and 30-day readmission following anterior cruciate ligament reconstruction. Knee Surg Sports Traumatol Arthrosc 2020;28:432-8.

27 Bokshan SL, DeFroda SF, Owens BD. Risk factors for hospital admission after anterior cruciate ligament reconstruction. Arthroscopy 2017;33:1405-11.

28 Eckenrode BJ, Carey JL, Sennett BJ, et al. Prevention and management of post-operative complications following $\mathrm{ACL}$ reconstruction. Curr Rev Musculoskelet Med 2017;10:315-21.

29 Agarwalla A, Gowd AK, Liu JN, et al. Effect of operative time on short-term adverse events after isolated anterior cruciate ligament reconstruction. Orthop J Sports Med 2019;7:232596711882545.

30 Kraus Schmitz J, Lindgren V, Janarv P-M, et al. Deep venous thrombosis and pulmonary embolism after anterior cruciate ligament reconstruction: incidence, outcome, and risk factors. Bone Joint $J$ 2019;101-B:34-40.

31 Muscolo DL, Carbo L, Aponte-Tinao LA, et al. Massive bone loss from fungal infection after anterior cruciate ligament arthroscopic reconstruction. Clin Orthop Relat Res 2009;467:2420-5.

32 Shamseer L, Moher D, Clarke M, et al. Preferred reporting items for systematic review and meta-analysis protocols (PRISMA-P) 2015: elaboration and explanation. BMJ 2015;349:g7647.

33 Stroup DF, Berlin JA, Morton SC, et al. Meta-Analysis of observational studies in epidemiology: a proposal for reporting. meta-analysis of observational studies in epidemiology (moose) group. JAMA 2000;283:2008-12.

34 Moher D, Liberati A, Tetzlaff J, et al. Preferred reporting items for systematic reviews and meta-analyses: the PRISMA statement. Ann Intern Med 2009;151:264-9.

35 Higgins J, Green S. Cochrane handbook for systematic reviews of interventions Version 5.1. 0 [updated March 2011]. London: The Cochrane Collaboration, 2016.

36 Wells GA, Shea B, O'Connell D, et al. The Newcastle-Ottawa scale (NOS) for assessing the quality of nonrandomized studies in meta-analysis. Available: http://www.ohri.ca/programs/clinical_ epidemiology/oxford.asp [Accessed 20 Feb 2020].

37 Jelicic Kadic A, Vucic K, Dosenovic S, et al. Extracting data from figures with software was faster, with higher interrater reliability than manual extraction. J Clin Epidemiol 2016;74:119-23.

38 PlotDigitizer software. Available: http://plotdigitizer.sourceforge.net/ [Accessed 20 Feb 2020].

39 DerSimonian R, Laird N. Meta-Analysis in clinical trials. Control Clin Trials 1986;7:177-88.

40 Higgins JPT, Thompson SG. Quantifying heterogeneity in a metaanalysis. Stat Med 2002;21:1539-58.

41 Harbord RM, Harris RJ, Sterne JAC. Updated tests for small-study effects in meta-analyses. Stata J 2009;9:197-210.

42 Egger M, Davey Smith G, Schneider M, et al. Bias in meta-analysis detected by a simple, graphical test. BMJ 1997;315:629-34.

43 Begg CB, Mazumdar M. Operating characteristics of a RANK correlation test for publication bias. Biometrics 1994;50:1088-101.

44 Wong ELY, Cheung AWL, Leung MCM, et al. Unplanned readmission rates, length of hospital stay, mortality, and medical costs of ten common medical conditions: a retrospective analysis of Hong Kong hospital data. BMC Health Serv Res 2011;11:149.

45 van Eck CF, Kropf EJ, Romanowski JR, et al. Acl graft re-rupture after double-bundle reconstruction: factors that influence the intra-articular pattern of injury. Knee Surg Sports Traumatol Arthrosc 2011;19:340-6.

46 Patterson BE, Culvenor AG, Barton CJ, et al. Worsening knee osteoarthritis features on magnetic resonance imaging 1 to 5 years after anterior cruciate ligament reconstruction. Am J Sports Med 2018;46:2873-83. 